\title{
Does a One Health Approach to Human African Trypanosomiasis Control Hasten Elimination? A Stochastic Compartmental Modeling Approach
}

Julianne Meisner ( $\square$ meisner.julianne@gmail.com )

University of Washington Seattle Campus: University of Washington https://orcid.org/0000-00017477-6598

\section{Agapitus Kato}

Uganda Virus Research Institute

Marshall Lemerani

Malawi Ministry of Health

\section{Erick Mwamba Miaka}

PNLTHA

\section{Acaga Ismail Taban}

IntraHealth: IntraHealth International Inc

Jonathan Wakefield

University of Washington Seattle Campus: University of Washington

\section{Ali Rowhani-Rahbar}

University of Washington Seattle Campus: University of Washington

\section{David Pigott}

University of Washington Seattle Campus: University of Washington Jonathan Mayer

University of Washington Seattle Campus: University of Washington

Christopher Lorton

IDM: Institute for Disease Modeling

\section{Peter Rabinowitz}

University of Washington Seattle Campus: University of Washington

\section{Research Article}

Keywords: human African trypanosomiasis, HAT, stochastic compartmental models, One Health, zoonoses

Posted Date: January 12th, 2022

DOI: https://doi.org/10.21203/rs.3.rs-1239807/v1 
License: (c) (i) This work is licensed under a Creative Commons Attribution 4.0 International License. Read Full License 


\title{
RESEARCH
}

\section{Does a One Health approach to human African trypanosomiasis control hasten elimination? A stochastic compartmental modeling approach}

\author{
Julianne Meisner ${ }^{1,2 *}$, Agapitus Kato ${ }^{3 \dagger}$, Marshall Lemerani ${ }^{4 \dagger}$, Erick M. Miaka ${ }^{5 \dagger}$, Acaga I. Taban ${ }^{6 \dagger}$, \\ Jonathan Wakefield ${ }^{10,11}$, Ali Rowhani-Rahbar ${ }^{1}$, David Pigott ${ }^{8}$, Jonathan D. Mayer ${ }^{1}$, Christopher \\ Lorton $^{7}$ and Peter M. Rabinowitz ${ }^{2,9}$
}

\footnotetext{
${ }^{*}$ Correspondence:

meisnerj@uw.edu

${ }^{2}$ Center for One Health Research, Department of Environmental and Occupational Health Sciences, University of Washington, Seattle, WA, USA

Full list of author information is available at the end of the article ${ }^{\dagger}$ Equal contributor
}

\begin{abstract}
Background: In response to large strides in the control of human African trypanosomiasis (HAT), in the early 2000s the WHO set targets for elimination of both the gambiense (gHAT) and rhodesiense (rHAT) forms as a public health (EPHP) problem by 2020, and elimination of gHAT transmisson (EOT) by 2030. While global EPHP targets have been met, and EOT appears within reach, there is ample evidence that current control strategies will not achieve gHAT EOT in the presence of animal reservoirs, the role of which is currently uncertain. Furthermore, rHAT is not targeted for EOT due to the known importance of animal reservoirs for this form.

Methods: To evaluate the utility of a One Health approach to gHAT and rHAT EOT, we built and parameterized a compartmental stochastic model, using the Institute for Disease Modeling's Compartmental Modeling Software, to six HAT epidemics: the national rHAT epidemics in Uganda and Malawi, the national gHAT epidemics in Uganda and South Sudan, and two separate gHAT epidemics in Democratic Republic of Congo distinguished by dominant vector species. In rHAT foci the reservoir animal sub-model was stratified on four species groups, while in gHAT foci domestic swine were assumed to be the only competent reservoir. The modeled time horizon was 2005-2045, with calibration performed using HAT surveillance data from 2000-2004 and Optuna. Interventions included insecticide and trypanocide treatment of domestic animal reservoirs at varying coverage levels.

Results: Validation against HAT surveillance data indicates favorable performance overall, with the possible exception of DRC. EOT was not observed in any modeled scenarios for rHAT, however insecticide treatment consistently performed better than trypanocide treatment in terms of rHAT control. EOT was not observed for gHAT at $0 \%$ coverage of domestic reservoirs with trypanocides or insecticides, but was observed by 2030 in all test scenarios; again, insecticides demonstrated superior performance to trypanocides.

Conclusions: EOT cannot be achieved for rHAT without control of wildlife reservoirs, however insecticide treatment of domestic animals holds promise for improved control. In the presence of domestic animal reservoirs, gHAT EOT will not be achieved under current control strategies.
\end{abstract}

Keywords: human African trypanosomiasis; HAT; stochastic compartmental models; One Health; zoonoses 


\section{Background}

Remarkable progress in the control of human African trypanosomiasis (HAT) in the early 2000s led the WHO to set targets for elimination as a public health problem (EPHP) by 2020, and elimination of transmission (EOT) by 2030. While EPHP goals include both the acute form of HAT, caused by Trypanosoma brucei rhodesiense (rHAT) and known to have important animal reservoirs, and the chronic form caused by $T$. b. gambiense (gHAT) and thought to be predominantly transmitted human-to-human, EOT goals target gHAT alone.

Mathematical modeling efforts for HAT have largely been deterministic, requiring the adoption of proxy thresholds for EOT (e.g., $<1$ new infection per 100,000 or 1,000,000 per year) [1] as such models represent populations with continuous variables that never reach zero [2]. Conversely, stochastic models evaluate the likelihood of disease elimination through natural failure of transmission events, allowing for representation of uncertainty and distribution of time to elimination [3]. Recently, there have been several stochastic efforts to model HAT elimination using Gillespie-based simulation algorithms [1]. Davis et al. (2019) used this approach, and a Ross-Macdonald-type stochastic compartmental model extended from Rock et al.'s (2015) previous deterministic model [4], to model gHAT persistence under varying parameterizations of reported screening patterns and population sizes at the village-level. They found probability of persistence increased with population size, but that gHAT transmission can persist for long periods in isolated populations as small as 2,000. Davis et al (2019) also found evidence of high spatial heterogeneity related to local environmental conditions, in particular proximity to large rivers [5]. Castaño et al. (2020) later extended two previously deterministic models to a stochastic framework, and found no strong evidence that current medical interventions and trends in decreasing case counts would be adequate to achieve EOT by 2030 in two health zones in DRC [6].

While multiple animal species harbor trypanosomes, their role in the transmission cycle is unclear [3]. Using data from Uganda, Davis et al. (2011) demonstrated that proportion of bloodmeals taken from humans was the most important determinant of gHAT distribution among those evaluated [7]. A model fit to animal prevalence data from Cameroon using the Next Generation Matrix approach and assuming a constant level of human infection suggested animals constitute a transmission reservoir for gHAT [8], while others fit to longitudinal human case data from Guinea, Chad, and DRC with decreasing reporting trends and time-varying interventions found equivocal support for presence versus absence of an animal reservoir $[4,9,10]$. These discrepancies may reflect differences in model assumptions, or the foci-specific nature of reservoir potential based on human-tsetse-animal abundance and contact patterns [1].

Two simple and cost-effective methods of vector control (VC) have emerged in the past decade: insecticide treatment of cattle (ITC) in the form of restricted application of synthetic pyrethroids where cattle are present in sufficient numbers (density $>10$ animals $/ \mathrm{km}^{2}$ ) and provide a significant proportion of bloodmeals for HAT vectors; and deployment of pyrethroid-treated tiny targets along the banks of rivers, the preferred habitat for riverine tsetse, otherwise [11]. Restricted application of synthetic pyrethroids to the belly and lower legs of older and larger cattle, the 
preferred feeding sites and hosts of HAT and animal African trypanosomiasis (AAT) vectors, respectively $[12,13,14]$, has been demonstrated to achieve $\mathrm{VC}$ at one tenth the cost of other ITC approaches (<US\$2/head/year [15]). In addition to minimizing cost and environmental residues, this approach contributes to control of AAT, widely considered to be the single greatest constraint to increased livestock production in Africa and an important poverty-reinforcing disease [16, 17, 18, 19]. In both gHAT and rHAT foci, ITC lowers the $R_{0}$ of HAT by reducing the average life expectancy of tsetse flies. In rHAT foci, ITC can further reduce $R_{0}$ by controlling the domestic animal reservoir of the disease and selectively killing tsetse infected by animal reservoirs, with potentially further gains being made through addition of trypanocide treatment (TT) of domestic animal reservoirs. Previous literature has demonstrated ITC is effective even when cattle are patchily distributed, with gaps of several kilometers wide [20].

In 2012, Hargrove et al. generalized Rogers (1988) [21] two-host deterministic compartmental model to study the effect of ITC and TT on the $R_{0}$ of rHAT. The authors found control of rHAT through TT alone is unlikely even if there are no wildlife reservoirs present. In contrast, ITC could eliminate rHAT if cattle comprise at least $40 \%$ of non-human tsetse bloodmeals and $100 \%$ of cattle are treated, or if cattle comprise $100 \%$ of non-human bloodmeals and $25 \%$ are treated [22]. While studies of effectiveness of ITC in gHAT foci are limited, spot-on ITC was found to reduce the fly population from 54.2 flies/trap/day to 0.06 flies/trap/day in a gHAT focus in Burkina Faso, with the remaining flies (Glossina palpalis palpalis) mainly feeding on monitor lizards, which are not thought to harbor human-infective trypanosomes [23].

As of 2018, eight out of 26 HAT-endemic countries were eligible for EPHP validation. We focus here on four countries who do not meet the required criteria for EPHP validation due to an excessive number of cases per health district (Malawi), inadequate control and surveillance activities (Uganda), or both (Democratic Republic of Congo (DRC) and South Sudan) [24]. In this study, we use a stochastic compartmental model implemented in the Institute for Disease Modeling's Compartmental Modeling Software (CMS) [25], to study the effect of including animal reservoirs in HAT control efforts under a One Health approach on time to EOT in these countries. We have parameterized this model to reflect the national epidemic in each country, fitting two models each for DRC and Uganda to reflect the presence of two gHAT vectors in DRC and the presence of both gHAT and rHAT in Uganda.

\section{Methods}

\section{Structure}

We constructed a stochastic compartmental of HAT transmission, implemented in CMS using the Gillespie algorithm [25], defining EOT as the first year after which no new transmission occurs for the remainder of the modeled time horizon. For gHAT we fit a four-species model with humans, tsetse flies, reservoir animals, and nonreservoir tsetse hosts (i.e., animal species from which tsetse flies take bloodmeals but which do not harbor human-infective trypanosomes), with domestic swine defined as the animal reservoir. For rHAT we fit a seven-species model with humans, tsetse flies, non-reservoir tsetse hosts, and four reservoir animal species groups: domestic 
swine, domestic bovids (cattle), wild swine (warthog), and wild bovids (bushbuck and African water buffalo).

Model structure is detailed in Figure 1. The sub-model is $\mathrm{S}-\mathrm{E}-\mathrm{I}_{1}-\mathrm{I}_{2}-\mathrm{R}$ for humans, S-E-I-R for reservoir animals, and S-E-I for flies with a non-susceptible compartment to reflect the teneral effect, whereby flies are most susceptible to transmission of trypanosomes during their first blood meal and within their first 24 hours of life. Humans with stage 2 illness are assumed to be inaccessible to flies and thus do not contribute to transmission. For the rHAT models, model structure is equivalent for the sub-model corresponding to each animal reservoir species group.

Time steps were one day, with an overall time horizon of 2005-2045. Previous stochastic compartmental models of HAT have indicated varying step size from 0.01 days to one day does not appreciably change results [5]. We assumed all populations are closed, that is a death is replaced by a susceptible, and no migration into or out of foci by infected flies, humans, or reservoir hosts occurs. Again, previous stochastic modeling efforts for HAT have demonstrated that such assumptions have negligible effects on model findings [5].

\section{Parameterization}

We parameterized this model to the national gHAT epidemics in South Sudan and Uganda; the national rHAT epidemics in Uganda and Malawi; and the Bandundu and Sakuru foci, together, and Equateur Nord focus, separately, in DRC. This parameterization, which resulted in a total of 6 models, was chosen to reflect the differences in the epidemiology of the each country's HAT epidemics, with the gHAT and rHAT epidemics being distinct within Uganda, and the Bandundu/Sankuru foci being distinct from the Equateur Nord focus in DRC due to different vector populations.

We assumed Glossina fuscipes fuscipes was the vector species in the Uganda gHAT, Uganda rHAT, South Sudan, and DRC Equateur Nord models. We assumed G. f. quanzensis was the vector species in the DRC Bandundu/Sakuru model, and G. morsitans morsitans in the Malawi model. For the human sub-models, we assumed all detected cases are treated, treatment is always successful, and no recovery occurs without treatment (i.e., cases transition from stage 2 infection to death). We assumed the population of non-reservoir tsetse hosts was stable throughout the modeled period.

Base-case model parameters are detailed in Additional file 1 - Model parameters. We derived parameters from published literature or model fitting, with the exception of the human:wild animal (reservoir or non-reservoir) ratio, which we took to be 10 in all cases; ITC coverage, which we took to be $0 \%$ for all reservoirs; TT coverage, which we took to be $0 \%$ for wild animal reservoirs in rHAT foci and domestic animal reservoirs in gHAT foci, and 50\% for domestic animal reservoirs in rHAT foci; and TT frequency, which we took to be every 3 months. Adapted from Davis et al. (2019), we defined effective tsetse density as the product of the fly:human ratio and $\beta_{H}$, the probability of human infection per single infective bite [5]. We fixed the former at 6.56, from Davis et al. (2019) in all models, but allowed $\beta_{H}$ to vary.

Model equations are presented in Additional file 2 - Model equations. 


\section{Interventions}

Insecticide treatment (ITC)

While ITC refers specifically to insecticide treatment of cattle, here we will model insecticide treatment of both cattle and pigs without modification to this term. As in Hargrove et al. (2012) [22], we assume ITC exerts its effect by decreasing the probability a fly survives a given feed, $q_{f}$. If $q_{n}$ is the probability a fly survives a nonfeeding day, and $d$ is the feeding cycle length, then the probability a fly survives a complete feeding cycle is $q_{f} q_{n}{ }^{d}$. As daily mortality rate is $\approx-\log \left(q_{f} q_{n}{ }^{d}\right) / d$, our parameterization of tsetse fly lifespan (26 days for G. m. morsitans and G. f. fuscipes, and 29 days for G. f. quanzensis) and feeding cycle length (3 days) yields $q_{f} q_{n}{ }^{d}=0.89$ for G. f. fuscipes and G. m. morsitans, and $q_{f} q_{n}{ }^{d}=0.91$ for $G$. $f$. quanzensis.

If $i t c_{R i}$ is the proportion of reservoir species $i$ treated with insecticides, $p_{R i}(t)$ is the probability a given bloodmeal is taken from reservoir species $i$ at time $t$, and flies feed on individual members of a given reservoir species at random, assuming efficacy of treatment is $100 \%$ the probability a fly survives a complete feeding cycle of $d$ days is now:

$$
\left[1-\sum_{i=1}^{I}\left(i t c_{R i} p_{R i}(t)\right)\right] q_{f} q_{n}^{d}
$$

Trypanocide treatment (TT)

Trypanocide treatment of domestic animal reservoirs was implemented through allowing a proportion of animals to receive trypanocidal treatment at three month intervals, thereby shortening their duration of infection. This is distinct from Hargrove's implementation of TT, which assumed continuous prophylactic use and therefore removal of a proportion of reservoirs from the reservoir population [22].

\section{Scenarios modeled}

We evaluated the probability of and time to EOT under the following scenarios:

- gHAT foci:

- ITC of pigs at $12.5 \%, 25 \%$ and $50 \%$ coverage

- ITC and TT of pigs at $25 \%$ coverage of each

- rHAT foci:

- ITC of cattle and pigs at $25 \%, 50 \%$, and $75 \%$ coverage

- ITC of cattle and pigs at $50 \%$ combined with TT of cattle and pigs at $75 \%$ coverage

- ITC and TT of cattle and pigs at $100 \%$ coverage of each

Our base-case model assumed $0 \%$ TT or ITC coverage in gHAT foci, and $50 \%$ TT coverage (both cattle and pigs) in rHAT foci. Note "cattle and pigs" refers to domestic bovids and domestic swine, respectively.

\section{Calibration}

We calibrated our models to 2000-2004 annual surveillance data from the WHO Atlas of HAT [26] using Optuna [27]. We collapsed observed data by country, keeping rHAT and gHAT foci separate in Uganda, and assumed $65 \%$ of gHAT cases are reported [28] and $8.3 \%$ of $\mathrm{rHAT}$ cases are reported [29]. 
Fitting 10 trials with 3 model runs per trial and optimizing the mean over these runs, we defined the sum of the squared error terms as the objective function to be minimized. Under normally-distributed and errors with constant variance, this simple objective function yields the same estimate as a maximum likelihood approach [30]. We used a tau-leaping solver and specified trial parameters as $\beta_{H} \sim$ Uniform $(0.001,0.1)$ for gHAT foci in Uganda and South Sudan, where $\beta_{H}$ is the probability an infected fly transmits to a human during a given bloodmeal; $\beta_{H} \sim$ Uniform $(0.001,0.01)$ for both gHAT foci in DRC, reflecting the markedly higher probability a bloodmeal is taken from a human (and thus force of infection) in these foci; and $\beta_{H} \sim \operatorname{Uniform}(0.0001,0.001)$ in rHAT foci.

\section{Validation}

We validated our model by comparing observed Atlas data to the predicted number of new cases per year in our base case model, adjusted for underreporting as detailed above. This was performed from 2005-2014 in Malawi and South Sudan, and from 2005-2018 in DRC and Uganda, reflecting the data made available to the authors.

\section{Results}

Model trajectories for tsetse flies and humans are presented in Additional file 3 - Model trajectories. While epidemiologic curves for humans vary in level across modeled scenarios, shape is relatively stable. In the rHAT models human infection is maintained at a remarkably low but steady level, consistent with epidemiologic evidence of HAT's ability to persist at low levels.

In contrast, the curves for tsetse flies vary markedly across modeled scenarios in the gHAT models, increasing (Uganda gHAT, DRC Bandundu/Sakuru) or reaching a steady-state (DRC Equateur Nord, South Sudan) in the base-case, versus decaying rapidly in all test scenarios. In the rHAT models, exposed and infected tsetse flip in the base-case versus test scenarios, with infected tsetse outnumbering exposed tsetse in the base-case, and the inverse being true in all test scenarios. This is consistent with ITC lowering the age distribution of tsetse flies.

\section{Validation}

Validation results are presented in Figure 2. On an absolute scale, the models appeared to perform better in later than earlier years, and performed well for Uganda in both gHAT and rHAT foci and for South Sudan and Malawi, but not very well in either DRC foci. However, the number of cases reported (observed) in each focus varies markedly (e.g., 608 total cases in Malawi and 16,756 total cases South Sudan over the period 2000-2014; and 4,219 total cases in Uganda gHAT foci, 3,281 total cases in Uganda rHAT foci, 68,414 total cases in the Bandundu/Sakuru foci in DRC, and 19,327 total cases in the Equateur Nord focus in DRC over the period 2000-2018), thus a difference between observed and predicted cases of 100 implies markedly different model performance in, for instance, Malawi versus either DRC foci.

\section{Elimination of transmission}

EOT outcomes under all models and scenarios are presented in Tables 1-2; predicted number of number of cases in 2030 for the base-case scenario in each modeled focus 
is also presented in Figure 3, and for all model scenarios in the rHAT foci (Uganda rHAT, Malawi) in Figures 4-5. Corresponding results are not presented for the gHAT models (Uganda gHAT, South Sudan, and both DRC models) as EOT was observed by 2030 all gHAT foci for all test scenarios.

Under current conditions, reflected by the base-case scenario, EOT is not observed by 2045 in any modeled focus, with cases being particularly high in Uganda and DRC across the two epidemics modeled in each country. For the gHAT models (Uganda gHAT, both DRC models, and South Sudan), EOT was observed by 2030 in all test scenarios, with differences in time to EOT across scenarios occurring on the order of months, not years (Table 1, Figures 6-9). Time to elimination is shortest for South Sudan, followed by Uganda and the Equateur Nord focus in DRC, and lastly by the Bandundu/Sakuru foci in DRC.

For both rHAT models (Uganda rHAT and Malawi), EOT is not observed in any test scenario. Case counts reduce monotonically with increasing ITC coverage, however increasing TT coverage from $50 \%$ to $75 \%$ has inferior gains to the same increase in ITC coverage (Table 2, Figures 4-5).

\section{Discussion}

We present the results of a stochastic compartmental model fit to six distinct HAT epidemics. Our findings indicate WHO EOT goals will not be met by 2030 under current conditions, however in gHAT foci in Uganda, South Sudan, and DRC, EOT would have already been achieved if insecticide treatment of domestic cattle and pigs had been implemented at even $12.5 \%$ coverage starting in 2005 . In rHAT foci in Uganda and Malawi, elimination of transmission is not achieved by the end of our modeled time horizon (2045) even if $100 \%$ of domestic cattle and pigs are constantly maintained on insecticide treatment with $100 \%$ efficacy, and receive $100 \%$-effective trypanocide treatment every three months. In both rHAT and gHAT foci, trypanocide treatment of domestic animal reservoirs hastens time to EOT (gHAT foci only) and reduces cases observed by 2030 (rHAT foci), but with inferior gains to increasing coverage with insecticide treatment of domestic animal resrevoirs.

Our model has several limitations. First, validation indicates our model predictions are a poor fit for both DRC models, suggesting results for this country should be interpreted in relative, rather than absolute, terms. Second, our results are sensitive to the assumptions made, namely detection perfectly predicts recovery in humans, TT and ITC are $100 \%$ effective, all populations are closed, and no inmigration of infected humans or tsetse occurs (i.e., each modeled focus is sufficiently isolated from other HAT foci). Previous authors have demonstrated the closed population and no in-migration assumptions have negligible impact on results in other stochastic compartmental HAT models [5].

Futhermore, while most parameters were derived from the literature or from model fitting, density of non-reservoir tsetse hosts was assumed, as was the probability domestic animal reservoirs receive trypanocide or insecticide treatment and frequency of the former. We also assumed tsetse flies have an inherent (species-specific) level of anthropophily, and all "remaining" bloodmeals (i.e., those taken from animals) are distributed according to density of each animal species or species group. 
The results of our test scenarios may also be sensitive to the way we implemented ITC and TT. Our implementation of TT assumes therapeutic rather than prophylactic use. With regards to ITC, our implementation assumes the tsetse population is constant such that increasing ITC coverage reduces the mean tsetse age -and thus the proportion infected with $T$. b. gambiense or $T$. b. rhodesiense-rather than density. Finally, we did not model vector control in the base-case scenario nor use of stationary baits in the test scenarios, nor any longitudinal change in active or passive surveillance coverage.

Our results indicate insecticide treatment of cattle and pigs should be added to control strategies in both gHAT and rHAT foci, however delivery of insecticides to pigs is slightly more challenging than cattle. First, deltamethrin, which is widelyavailable in many HAT-endemic areas and has a long duration of action, is not labeled for pigs, however it has been successfully used off-label to control biting flies and mosquitoes in Australia [31]. Second, while permethrin may be a suitable alternative, the efficacy of restricted application approaches have not been evaluated in pigs, and efficacy of alternative modes of delivery is uncertain. Possible delivery options include bi-weekly sprays, use of back rubbers, or impregnated fabrics placed near pigs but out of their reach. Third, the effectiveness of using pigs as live baits has not been established, however their proclivity for roaming in shady areas along the riverine habitats favored by HAT vectors [32] is a favorable indication in this regard.

\section{Conclusion}

Despite the limitations of our model, and potential challenges to implementation of the results it points to, our approach nonetheless represents an important contribution to the HAT modeling literature. This is, to our knowledge, the first effort use a stochastic compartmental model to study the utility of a One Health approach to HAT control across foci representing distinct epidemiologic, entomologic, and environmental conditions. By harmonizing model structure and assumptions, our study increases the comparability of results across these foci. Our results confirm the widely-held belief that elimination of rHAT transmission will not occur even at complete coverage of domestic animal reservoirs with trypanocides and insecticides, and indicate that if pigs are a reservoir of gHAT, EOT goals can only be obtained if insecticide or trypanocide treatment of this reservoir host is added to HAT control strategies, with insecticide treatment being superior. In addition to increasing the speed and probability of gHAT EOT, and contributing to control of rHAT, coordinated top-down implementation of joint HAT-AAT control strategies hold opportunity to retain donor engagement in HAT as gHAT elimination nearspreventing gHAT re-emergence due to animal reservoirs or latent human infections, and rHAT emergence as a major public health problem - and to contribute to the control of an important and poverty-reinforcing veterinary disease.

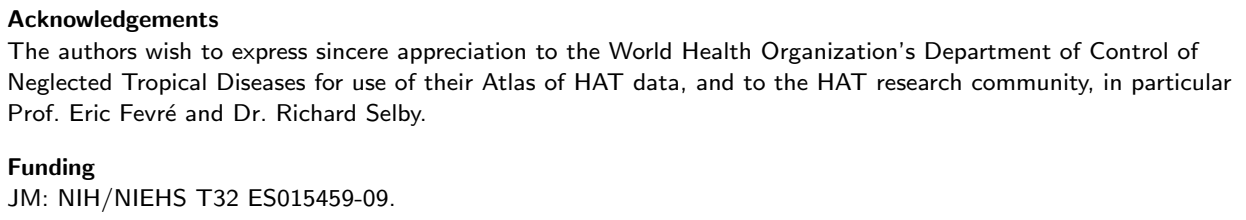




\section{Abbreviations}

AAT: Animal African trypanosomiasis

CMS: Compartmental modeling software

EOT: Elimination of transmission

EPHP: Elimination as a public health problem

DRC: Democratic Republic of Congo

gHAT: Gambiense (chronic) human African trypanosomiasis

HAT: Human African trypanosomiasis

ITC: Insecticide treatment of cattle and pigs

rHAT: Rhodesiense (acute) human African trypanosomiasis

TT: Trypanocide treatment of cattle and pigs

VC: Vector control

WHO: World Health Organization

\section{Availability of data and materials}

HAT outcome data can be requested from the WHO

(https://www. who.int/trypanosomiasis_african/country/foci_AFRO/en/) and livestock density maps can be downloaded from https://github.com/JulianneMeisnerUW/LivestockMaps. Parameter data can be obtained from the references detailed in Additional file 1 - Model parameters.

CMS can be downloaded from https://docs.idmod.org/projects/cms/en/latest/index.html\#, and model code written in Python is available in the GitHub repository linked above.

\section{Ethics approval and consent to participate}

This study used only routinely-collected surveillance data, which did not contain any individual-level identifiers, and publicly-available datasets. Thus, it does not constitute human subjects research.

\section{Competing interests \\ The authors declare that they have no competing interests.}

Consent for publication

Not applicable.

\section{Authors' contributions}

$\mathrm{JM}$ : validation (design, regression and prediction models), mapping, manuscript (initial and subsequent drafts). AK, ML, EMM, AIT: data acquisition, manuscript revisions. JW: design, conceptualization, manuscript revisions. ARR: design, manuscript revisions. DP: design, conceptualization, manuscript revisions. JDM: conceptualization, manuscript revisions. CL: software, design. PMR: design, conceptualization, supervision, manuscript revisions.

\section{Author details}

${ }^{1}$ Department of Epidemiology, University of Washington, Seattle, WA, USA. ${ }^{2}$ Center for One Health Research, Department of Environmental and Occupational Health Sciences, University of Washington, Seattle, WA, USA.

${ }^{3}$ Ministry of Agriculture, Animal Industry and Fisheries, Entebbe, Uganda. ${ }^{4}$ Trypanosomiasis Control Programme, Ministry of Health, Lilongwe, Malawi. ${ }^{5}$ Programme National de Lutte contre la Trypanosomiase Humaine Africaine, Kinshasa, DRC. ${ }^{6}$ IntraHealth International, Juba, South Sudan. ${ }^{7}$ Institute for Disease Modeling, Seattle, WA, USA. ${ }^{8}$ Institute for Health Metrics and Evaluation, University of Washington, Seattle, WA, USA. ${ }^{9}$ Department of Global Health, University of Washington, Seattle, WA, USA. ${ }^{10}$ Department of Statistics, University of Washington, Seattle, WA, USA. ${ }^{11}$ Department of Biostatistics, University of Washington, Seattle, WA, USA.

\section{References}

1. Review, O.P., Status, R.: Insights from quantitative and mathematical modelling on the proposed 2030 goal for gambiense human African trypanosomiasis (gHAT) [version 2; peer review: 2 approved] NTD Modelling Consortium Discussion Group on Gambiense Human African Gates Open R, 1-16 (2020)

2. Aliee, M., Rock, K.S.., Keeling, M.J..: Estimating the distribution of time to extinction of infectious diseases in mean-field approaches. Journal of the Royal Society, Interface 17(173), 20200540 (2020). doi:10.1098/rsif.2020.0540

3. Rock, K.S., Ndeffo-Mbah, M.L., Castaño, S., Palmer, C., Pandey, A., Atkins, K.E., Ndung'U, J.M., Hollingsworth, T.D., Galvani, A., Bever, C., Chitnis, N., Keeling, M.J.: Assessing strategies against gambiense sleeping sickness through mathematical modeling. Clinical Infectious Diseases 66(Suppl 4), 286-292 (2018). doi:10.1093/cid/ciy018

4. Rock, K.S., Torr, S.J., Lumbala, C., Keeling, M.J.: Quantitative evaluation of the strategy to eliminate human African trypanosomiasis in the Democratic Republic of Congo. Parasites and Vectors 8(1), 1-13 (2015). doi:10.1186/s13071-015-1131-8

5. Davis, C.N., Rock, K.S., Miaka, E.M., Keeling, M.J.: Village-scale persistence and elimination of gambiense human African trypanosomiasis. PLoS Neglected Tropical Diseases 13(10), 1-15 (2019). doi:10.1371/journal.pntd.0007838

6. Castaño, M.S., Aliee, M., Mwamba Miaka, E., Keeling, M.J., Chitnis, N., Rock, K.S.: Screening Strategies for a Sustainable Endpoint for Gambiense Sleeping Sickness. Journal of Infectious Diseases 221, 539-545 (2020). doi:10.1093/infdis/jiz588

7. Davis, S., Aksoy, S., Galvani, A.: A global sensitivity analysis for African sleeping sickness. Parasitology 138(4), 516-526 (2011). doi:10.1017/S0031182010001496

8. Funk, S., Nishiura, H., Hesterbeek, H., Edmunds, W.J., Checchi, F.: Identifying transmission cycles at the human-animal interface: the role of animal reservoirs in maintaining gambiense human African trypanosomiasis. PLoS Comput Biol 9(1), 1002855 (2013). doi:10.1371/journal.pcbi.1002855 
9. Pandey, A., Atkins, K.E., Bucheton, B., Camara, M., Aksoy, S., Galvani, A.P., Ndeffo-Mbah, M.L.: Evaluating long-term effectiveness of sleeping sickness control measures in Guinea. Parasites and Vectors 8, 550 (2015). doi:10.1186/s13071-015-1121-x

10. Mahamat, M.H., Peka, M., Rayaisse, J.B., Rock, K.S., Toko, M.A., Darnas, J., Brahim, G.M., Alkatib, A.B., Yoni, W., Tirados, I., Courtin, F., Brand, S.P.C., Nersy, C., Alfaroukh, I.O., Torr, S.J., Lehane, M.J., Solano, P.: Adding tsetse control to medical activities contributes to decreasing transmission of sleeping sickness in the Mandoul focus (Chad). PLoS Neg Trop Dis 11(7), 0005792 (2017). doi:e0005792

11. Tirados, I., Hope, A., Selby, R., Mpembele, F., Miaka, E.M., Boelaert, M., Lehane, M.J., Torr, S.J., Stanton, M.C.: Impact of tiny targets on Glossina fuscipes quanzensis, the primary vector of human African trypanosomiasis in the Democratic Republic of the Congo. PLOS Neglected Tropical Diseases 14, 0008270 (2020). doi:10.1371/journal.pntd.0008270

12. Vale, G.A., Mutika, G., Lovemore, D.F.: Insecticide-treated cattle for controlling tsetse flies (Diptera: Glossinidae): some questions answered, many posed. Bulletin of Entomological Research 89, 569-578 (1999)

13. Torr, S.J., Hargrove, W.: Factors affecting the landing and feeding responses of the tsetse fly Glossina pallidipes to a stationary ox. Medical and Veterinary Entomology 12, 196-207 (1998)

14. Torr, S.J., Wilson, P.J., Schofield, S., Mangwiro, T.N.C., Akber, S., White, B.N.: Application of DNA markers to identify the individual-specific hosts of tsetse feeding on cattle. Medical and Veterinary Entomology 15, 78-86 (2001)

15. Shaw, A.P.M., Torr, S.J., Waiwa, C., Cecchi, G., Wint, G.R.W., Mattioli, R.C., Robinson, T.P.: Estimating the costs of tsetse control options: an example for Uganda. Preventative Veterinary Medicine 110, 290-303 (2013)

16. Verysen, M.J.B.: Prospects for area-wide integrated control of tsetse flies (Diptera: Glossinidae) and trypanosomosis in sub-Saharan Africal. Rev Soc Entomol Argent 65, 1-21 (2006)

17. Alsan, M.: The effect of the tsetse fly on African development. American Economic Review 105(1), 382-410 (2015)

18. Duguma, R., Tasew, S., Olani, A., Damena, D., Alemu, D., Mulatu, T., Alemayehu, Y., Yohannes, M., Bekana, M., Hoppenheit, A., Abatih, E., Habtewold, T., Delespaux, V., Duchateau, L.: Spatial distribution of Glossina sp. and Trypanosoma sp. in south-western Ethiopia. Parasites and Vectors 8(430) (2015). doi:10.1186/s13071-015-1041-9

19. Rogers, D.J., Randolph, S.E.: Tsetse flies in Africa: bane or boon? Conservation Biology 2(1), 57-65 (1988)

20. Torr, S.J., Vale, G.A.: Is the even distribution of insecticide-treated cattle essential for tsetse control? Modelling the impact of baits in heterogeneous environments. PLoS Neg Trop Dis 5(10), 1360 (2011)

21. Rogers, D.J.: A general model for the African trypanosomiases. Parasitology 97(1), 193-212 (1988). doi:10.1017/S0031182000066853

22. Hargrove, J.W., Ouifki, R., Kajunguri, D., Vale, G.A., Torr, S.J.: Modeling the control of trypanosomiasis using trypanocides or insecticide-treated livestock. PLoS Neg Trop Dis 6(5), 1615 (2012). doi:10.1371/journal.pntd.0001615

23. Bauer, B., Amsel-de Lafosse, S., Calusen, P.H., Kabore, I., Petrich-Bauer, J.: Successful application of deltamethrin pour on to cattle in a campaign against tsetse flies (Glossina spp.) in the pastoral zone of Samorogouan, Burkina Faso. Trop Med Parasitol 36(3), 183-9 (1995)

24. Franco, J.R., Cecchi, G., Priotto, G., Paone, M., Diarra, A., Grout, L., Simarro, P.P., Zhao, W., Argaw, D.: Monitoring the elimination of human African trypanosomiasis at continental and country level: Update to 2018. PLoS Neglected Tropical Diseases 14(5), 1-18 (2020). doi:10.1371/journal.pntd.0008261

25. Lorton, C.W., Proctor, J.L., Roh, M.K., Welkhoff, P.A.: Compartmental Modeling Software: A fast, discrete stochastic framework for biochemical and epidemiological simulation. bioRxiv (2019). doi:10.1101/609172

26. Simarro, P.P., Cecchi, G., Paone, M., Franco, J.R., Diarra, A., Ruiz, J.A., Fevre, E.M., Courtin, F., Mattioli, R.C., Jannin, J.G.: The Atlas of human African trypanosomiasis: a contribution to global mapping of neglected tropical diseases. International Journal of Health Geographics 9, 57 (2010)

27. Akiba, T., Sano, S., Yanase, T., Ohta, T., Koyama, M.: Optuna: A next-generation hyperparameter optimization framework. In: Proceedings of the 25rd ACM SIGKDD International Conference on Knowledge Discovery and Data Mining (2019)

28. Checchi, F., Cox, A.P., Chappuis, F., Priotto, G., Chandramohan, D., Haydon, D.T.: Prevalence and under-detection of gambiense human African trypanosomiasis during mass screening sessions in Uganda and Sudan. Parasites \& Vectors 5(157) (2012). doi:10.1186/1756-3305-5-157

29. Odiit, M., Coleman, P.G., Liu, W.C., McDermott, J.J., Fèvre, E.M., Welburn, S.C., Woolhouse, M.E.: Quantifying the level of under-detection of Trypanosoma brucei rhodesiense sleeping sickness cases. Trop Med Int Health 10(9), 840-9 (2005). doi:10.1111/j.1365-3156.2005.01470.x

30. A. King. Introduction to inference: parameter estimation. https://kingaa.github.io/short-course/parest/parest.html. Accessed 1 Mar 2021

31. Control of Biting Insects on Pigs. The Pig Site. https://www.thepigsite.com/articles/control-of-biting-insects-on-pigs. Accessed 26 Feb 2021

32. N'Djetchi, M.K., Ilboudo, H., Koffi, M., Kaboré, J., Kaboré, J.W., Kaba, D., Courtin, F., Coulibaly, B., Fauret, P., Kouakou, L., Ravel, S., Deborggraeve, S., Solano, P., De Meeûs, T., Bucheton, B., Jamonneau, V.: The study of trypanosome species circulating in domestic animals in two human African trypanosomiasis foci of Côte d'Ivoire identifies pigs and cattle as potential reservoirs of Trypanosoma brucei gambiense. PLoS Negl Trop Dis 11(10), 0005993 (2017). doi:10.1371/journal.pntd.0005993

Figures

Tables 
Figure 1: Schematic of stochastic compartmental model. Dashed lines correspond to transmission events; non-reservoir tsetse hosts do not contribute to transmission. S: susceptible; E: exposed; $\mathrm{I}_{1}$ : infected stage $1 ; \mathrm{I}_{2}$ : infected stage $2 ; \mathrm{R}$ : recovered; NS: non-susceptible.

\section{(a) Uganda gHAT}

(b) DRC, Equateur Nord focus
(c) DRC, Bandundu/Sakuru foci
(d) South Sudan
(e) Uganda rHAT
(f) Malawi

Figure 2: Observed minus predicted cases by year over 10 model runs in the base-case scenario
(a) Uganda gHAT
(b) DRC, Equateur Nord focus

(c) DRC, Bandundu/Sakuru foci

(d) South Sudan

(e) Uganda rHAT

(f) Malawi

Figure 3: Predicted HAT cases in 2030 over 10 model runs in the base-case scenario
(a) Base case
(b) $25 \%$ ITC
(c) $50 \%$ ITC
(d) $75 \%$ ITC
(e) $75 \%$ TT, $50 \%$ ITC
(f) $100 \% \mathrm{TT}, 100 \%$ ITC

Figure 4: Predicted cases in 2030, Uganda rHAT, over 10 runs. ITC: insecticide treatment of cattle and pigs; TT: trypanocide treatment of cattle and pigs. TT coverage assumed to be $50 \%$ unless otherwise specified
(a) Base case
(b) $25 \%$ ITC
(c) $50 \%$ ITC
(d) $75 \%$ ITC

(e) $75 \%$ TT, $50 \%$ ITC

(f) $100 \%$ TT, $100 \%$ ITC

Figure 5: Predicted cases in 2030, Malawi, over 10 runs. ITC: insecticide treatment of cattle and pigs; TT: trypanocide treatment of cattle and pigs. TT coverage assumed to be $50 \%$ unless otherwise specified.

Additional file 2 - Model equations

A .pdf file containing all model equations.

Additional file 3 - Model trajectories

A .pdf file containing key human and tsetse fly trajectories as figures. 

(a) Base case
(b) $12.5 \%$ ITC
(c) $25 \%$ ITC
(d) $50 \%$ ITC

(e) $25 \% \mathrm{TT}, 25 \%$ ITC

Figure 6: Predicted year of gHAT elimination, Uganda, over 10 runs. ITC: insecticide treatment of pigs; TT: trypanocide treatment of pigs. TT coverage assumed to be $0 \%$ unless otherwise specified. Dashed vertical line marks 2030 (WHO target year for gHAT EOT)
(a) Base case
(b) $12.5 \%$ ITC
(c) $25 \%$ ITC
(d) $50 \%$ ITC

(e) $25 \%$ TT, $25 \%$ ITC

Figure 7: Predicted year of gHAT elimination, DRC Equateur Nord focus, over 10 runs. ITC: insecticide treatment of pigs; TT: trypanocide treatment of pigs. TT coverage assumed to be $0 \%$ unless otherwise specified. Dashed vertical line marks 2030 (WHO target year for gHAT EOT)
(a) Base case
(b) $12.5 \%$ ITC
(c) $25 \%$ ITC
(d) $50 \%$ ITC

(e) $25 \% \mathrm{TT}, 25 \%$ ITC

Figure 8: Predicted year of gHAT elimination, DRC Bandundu/Sakuru foci, over 10 runs. ITC: insecticide treatment of pigs; TT: trypanocide treatment of pigs. TT coverage assumed to be $0 \%$ unless otherwise specified. Dashed vertical line marks 2030 (WHO target year for gHAT EOT)
(a) Base case
(b) $12.5 \%$ ITC
(c) $25 \%$ ITC
(d) $50 \%$ ITC

(e) $25 \%$ TT, $25 \%$ ITC

Figure 9: Predicted year of gHAT elimination, South Sudan, over 10 runs. ITC: insecticide treatment of pigs; TT: trypanocide treatment of pigs. TT coverage assumed to be $0 \%$ unless otherwise specified. Dashed vertical line marks 2030 (WHO target year for gHAT EOT)

Additional file 4 - Livestock mapping manuscript

A .pdf file of the manuscript containing detail on methodology for livestock mapping. 
Table 1: Results for gHAT models across 10 runs per model

\begin{tabular}{lcr}
\hline Model & EOT Year, mean (sd) & Cases, 2030, mean (sd) \\
\hline \multicolumn{3}{c}{ Uganda, gHAT } \\
\hline Base-case & NA & $106.50(11.54)$ \\
$12.5 \%$ ITC & $2010(1.89)$ & $0(0)$ \\
$25 \%$ ITC & $2010(1.32)$ & $0(0)$ \\
$25 \%$ TT, 25\% ITC & $2010(1.89)$ & $0(0)$ \\
$50 \%$ ITC & $2009(1.35)$ & $0(0)$ \\
\hline \multicolumn{3}{c}{ NTC, Equateur Nord focus } \\
\hline Base-case & NA & $88.60(10.77)$ \\
$12.5 \%$ ITC & $2010(1.77)$ & $0(0)$ \\
$25 \%$ ITC & $2010(2.02)$ & $0(0)$ \\
$50 \%$ ITC & $2010(1.71)$ & $0(0)$ \\
$25 \%$ TT, 25\% ITC & $2010(1.99)$ & $0(0)$ \\
\hline & NRA & \\
\hline Base-case & DRC, Bandundu/Sakuru foci & $0(0)$ \\
$12.5 \%$ ITC & $2013(1.23)$ & $0(0)$ \\
$25 \%$ ITC & $2013(1.84)$ & $0(0)$ \\
$50 \%$ ITC & $2013(1.17)$ & $0(0)$ \\
$25 \%$ TT, 25\% ITC & $2013(1.60)$ & \\
\hline & South Sudan & $0(0)$ \\
\hline Base-case & NA & $0(0)$ \\
$12.5 \%$ ITC & $2009(1.57)$ & $0(0)$ \\
$25 \%$ ITC & $2009(1.83)$ & $0(0)$ \\
$50 \%$ ITC & $2009(1.32)$ & \\
$25 \%$ TT, 25\% ITC & $2008(1.52)$ & \\
\hline
\end{tabular}

EOT: eliminaton of transmission; ITC: insecticide treatment of pigs; TT: trypanocide treatment of pigs; NA: eliminaton not observed. TT coverage is assumed to be $0 \%$ unless otherwise specified

Table 2: Results for rHAT models across 10 runs per model

\begin{tabular}{|c|c|c|}
\hline Model & EOT Year, mean (sd) & Cases, 2030, mean (sd) \\
\hline \multicolumn{3}{|c|}{ Uganda, rHAT } \\
\hline Base-case & NA & $86.20(11.56)$ \\
\hline $25 \%$ ITC & NA & $34.30(5.96)$ \\
\hline $50 \%$ ITC & NA & $19.10(4.79)$ \\
\hline $75 \%$ ITC & NA & $9.80(1.69)$ \\
\hline $75 \%$ TT, $50 \%$ ITC & NA & $16.60(3.44)$ \\
\hline $100 \%$ TT, $100 \%$ ITC & NA & $4.90(2.33)$ \\
\hline \multicolumn{3}{|c|}{ Malawi } \\
\hline Base-case & NA & $12.10(2.33)$ \\
\hline $25 \%$ ITC & NA & $3.50(0.85)$ \\
\hline $50 \%$ ITC & NA & $2.90(1.66)$ \\
\hline $75 \%$ ITC & NA & $1.80(1.32)$ \\
\hline $75 \%$ TT, $50 \%$ ITC & NA & $2.30(1.42)$ \\
\hline $100 \%$ TT, $100 \%$ ITC & NA & $0.80(1.03)$ \\
\hline
\end{tabular}

TT: trypanocide treatment of cattle and pigs; NA: elimination not observed. TT coverage is assumed to be $50 \%$ unless otherwise specified 
Figures

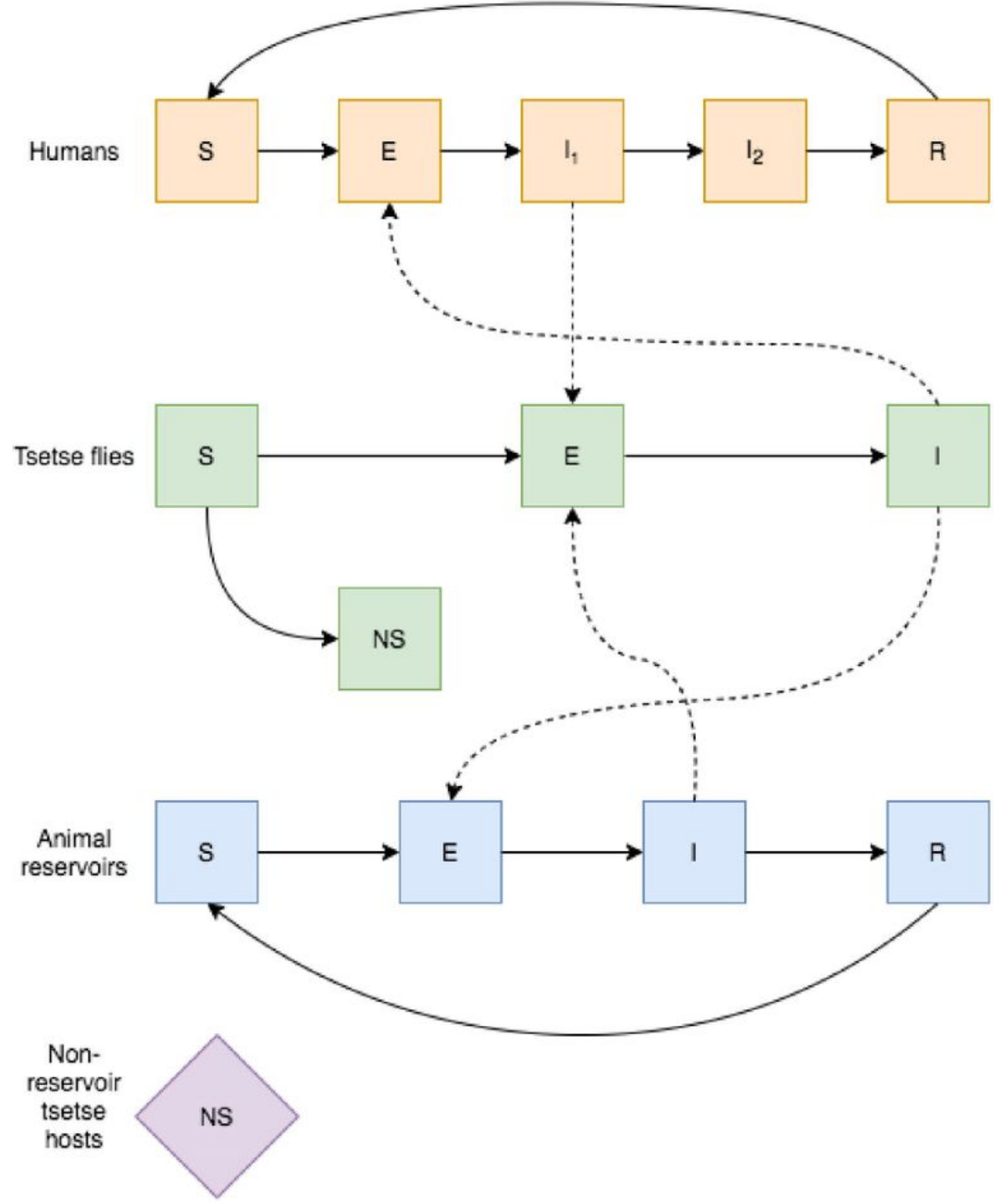

Figure 1: Schematic of stochastic compartmental model. Dashed lines correspond to transmission events; non-reservoir tsetse hosts do not contribute to transmission. S: susceptible; E: exposed; $\mathrm{I}_{1}$ : infected stage $1 ; \mathrm{I}_{2}$ : infected stage 2; R: recovered; NS: nonsusceptible.

\section{Figure 1}

Please See image above for figure legend. 


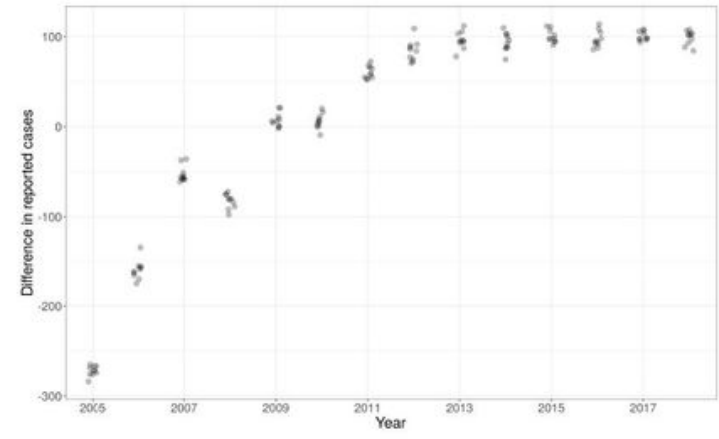

(a) Uganda gHAT

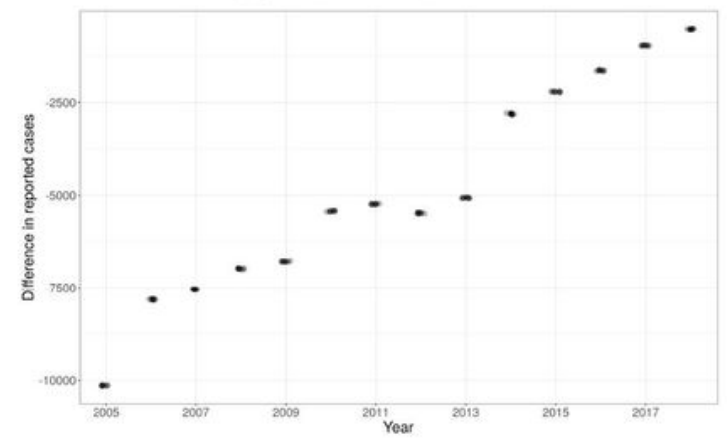

(c) DRC, Bandundu/Sakuru foci

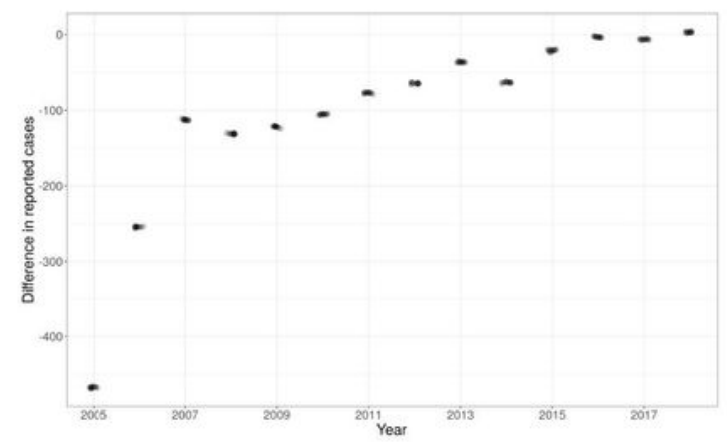

(e) Uganda rHAT

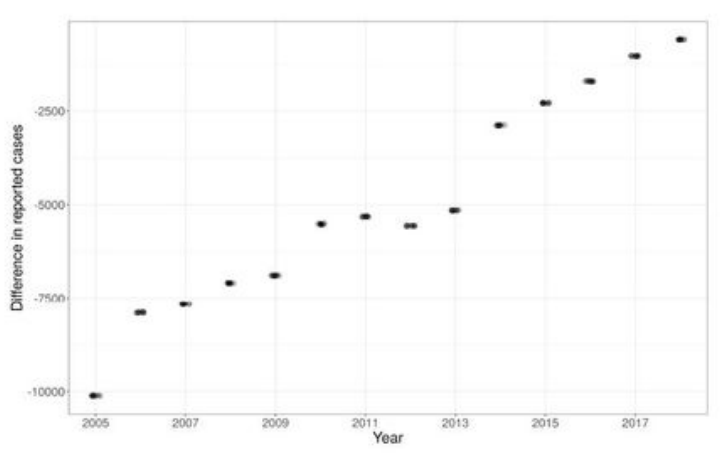

(b) DRC, Equateur Nord focus

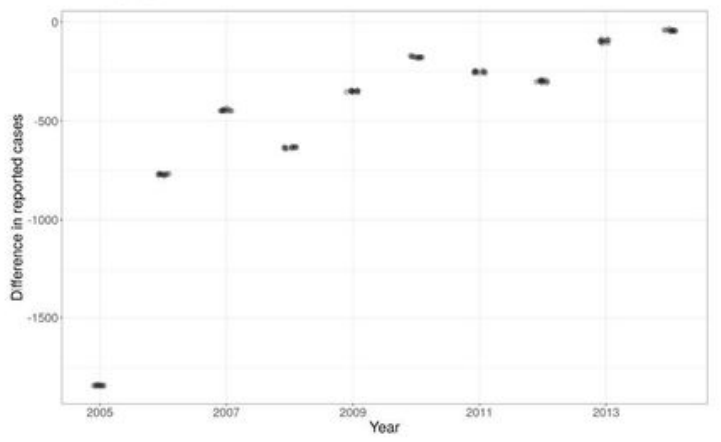

(d) South Sudan

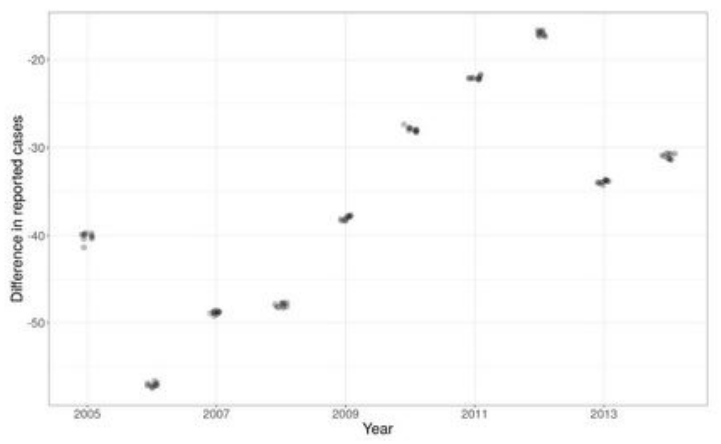

(f) Malawi

Figure 2: Observed minus predicted cases by year over 10 model runs in the base-case scenario

\section{Figure 2}

Please See image above for figure legend. 


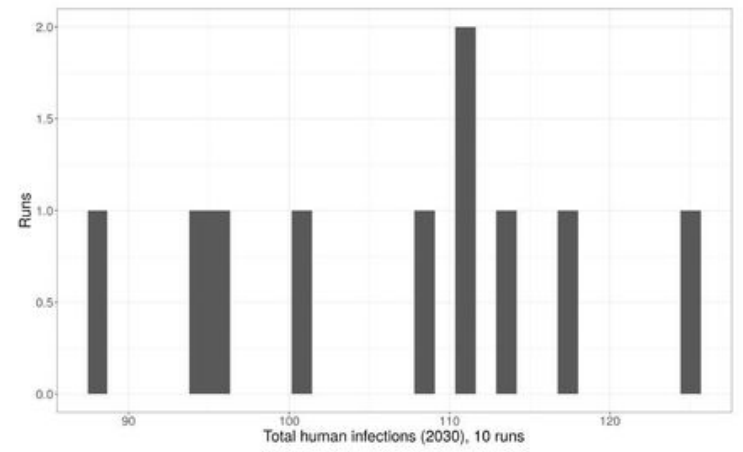

(a) Uganda gHAT

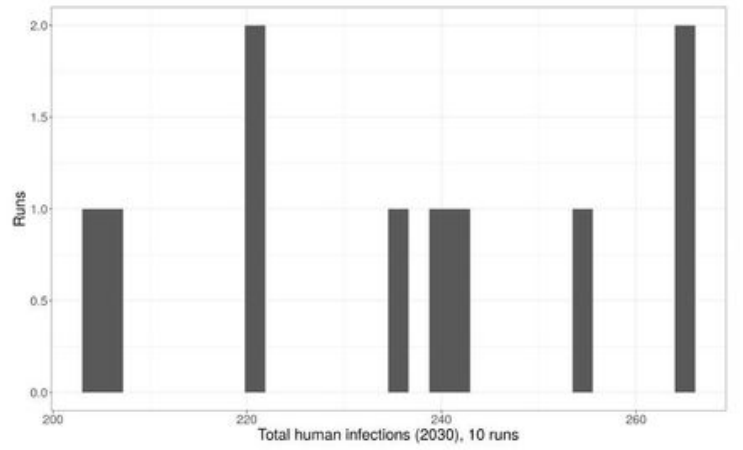

(c) DRC, Bandundu/Sakuru foci

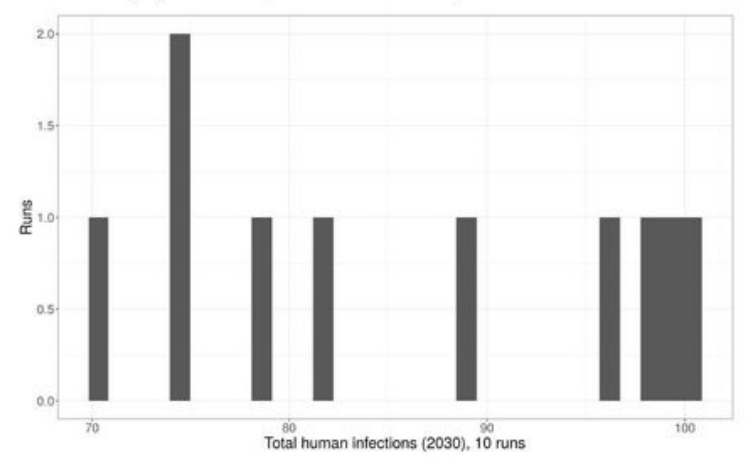

(e) Uganda rHAT

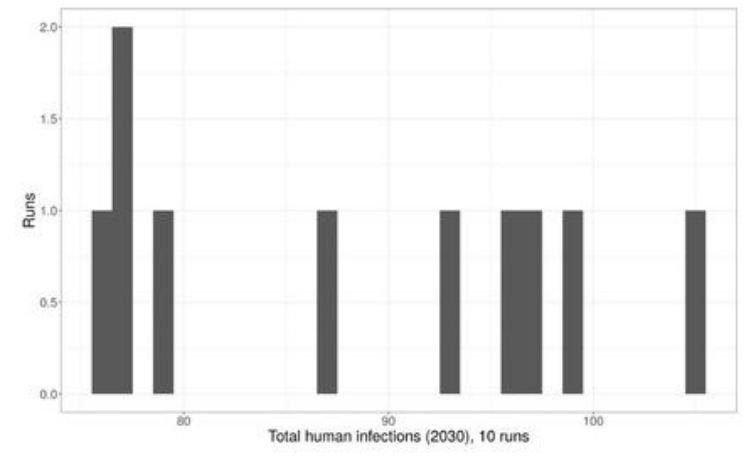

(b) DRC, Equateur Nord focus

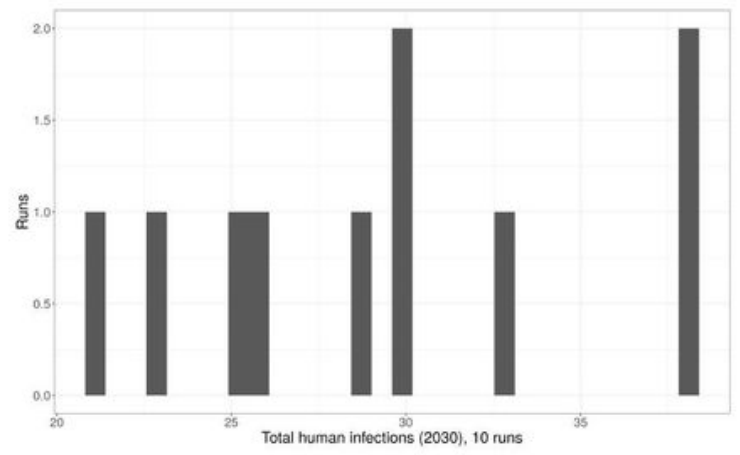

(d) South Sudan

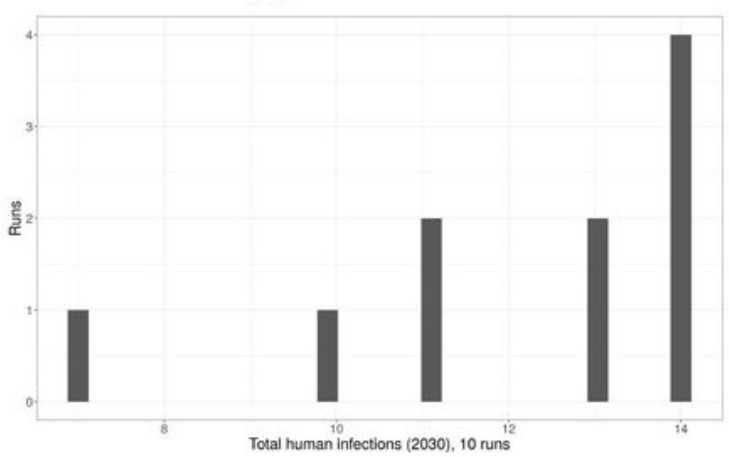

(f) Malawi

Figure 3: Predicted HAT cases in 2030 over 10 model runs in the base-case scenario

Figure 3

Please See image above for figure legend. 


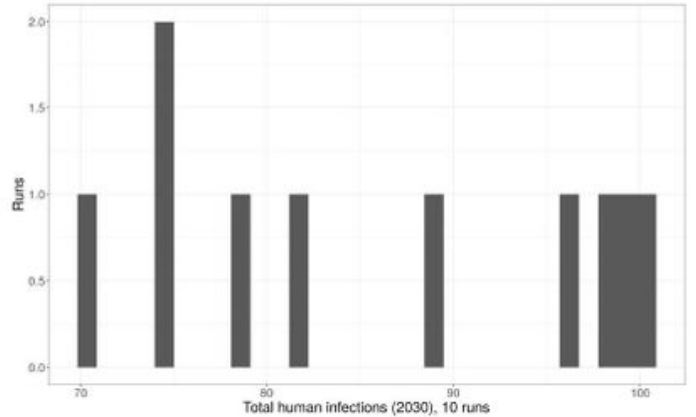

(a) Base case

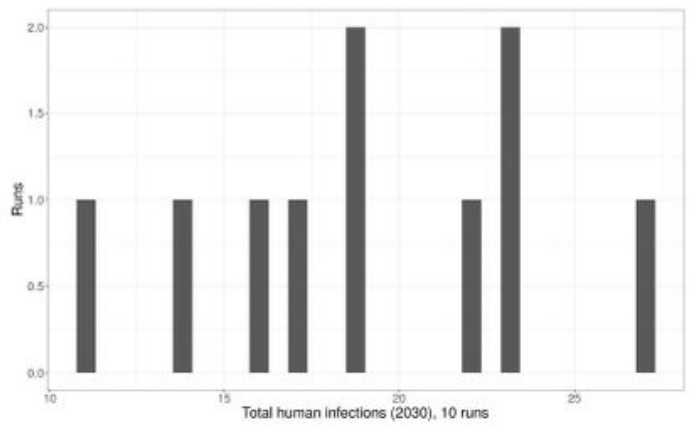

(c) $50 \%$ ITC

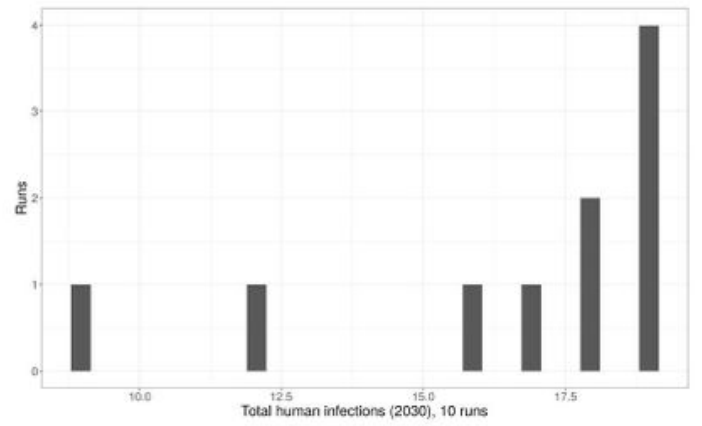

(e) $75 \%$ TT, $50 \%$ ITC

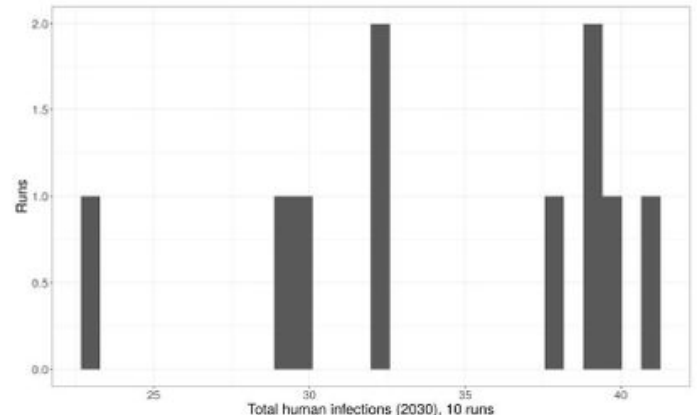

(b) $25 \%$ ITC

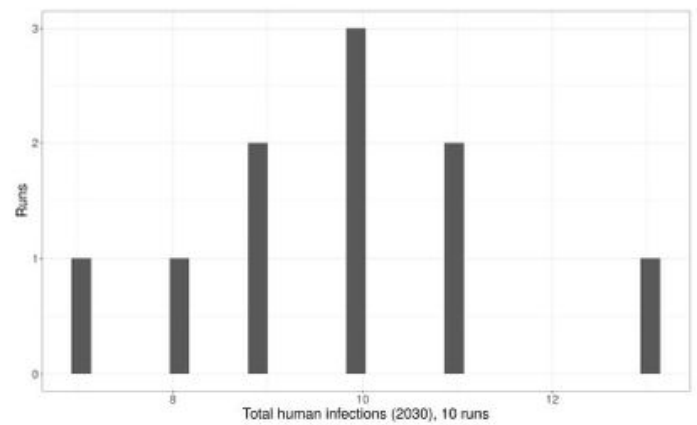

(d) $75 \%$ ITC

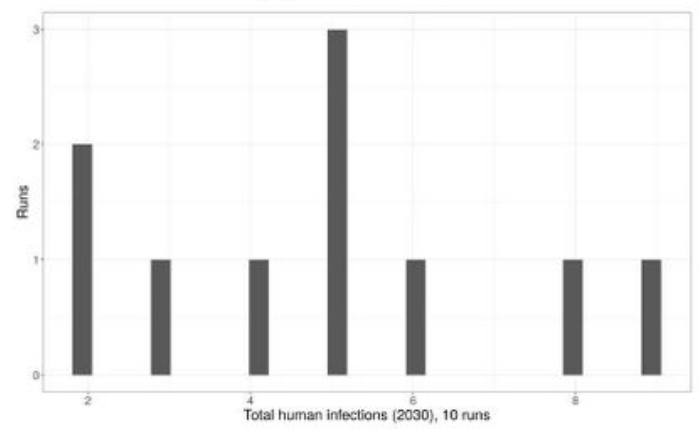

(f) $100 \% \mathrm{TT}, 100 \% \mathrm{ITC}$

Figure 4: Predicted cases in 2030, Uganda rHAT, over 10 runs. ITC: insecticide treatment of cattle and pigs; TT: trypanocide treatment of cattle and pigs. TT coverage assumed to be $50 \%$ unless otherwise specified

Figure 4

Please See image above for figure legend. 


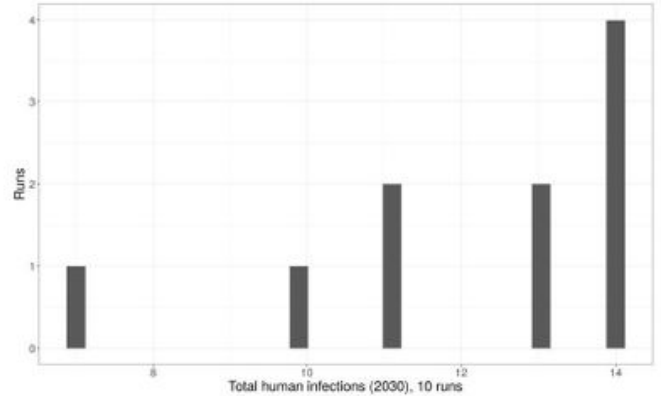

(a) Base case

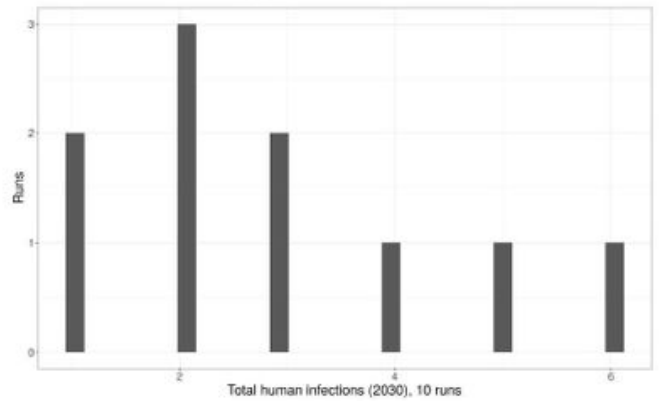

(c) $50 \%$ ITC

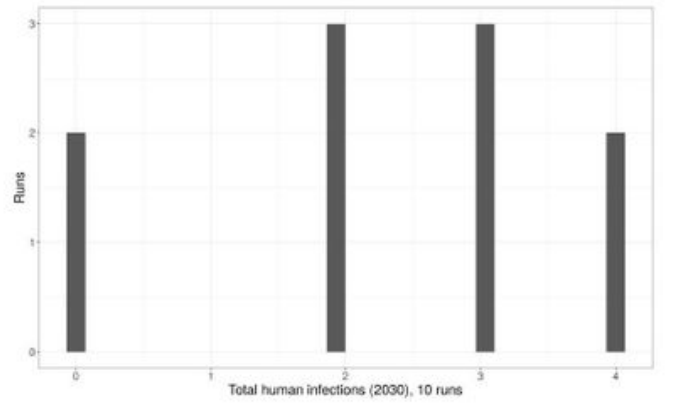

(e) $75 \%$ TT, $50 \%$ ITC

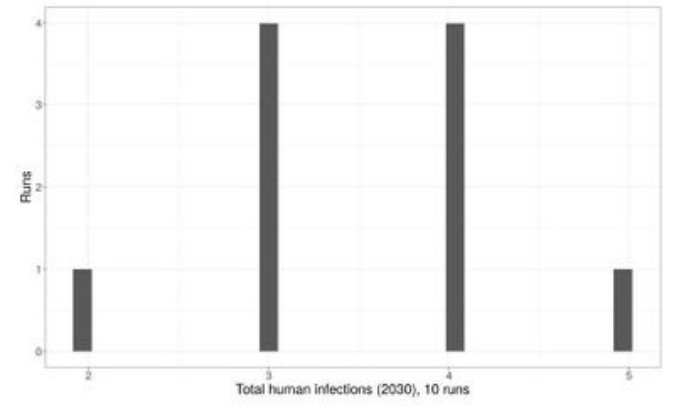

(b) $25 \%$ ITC

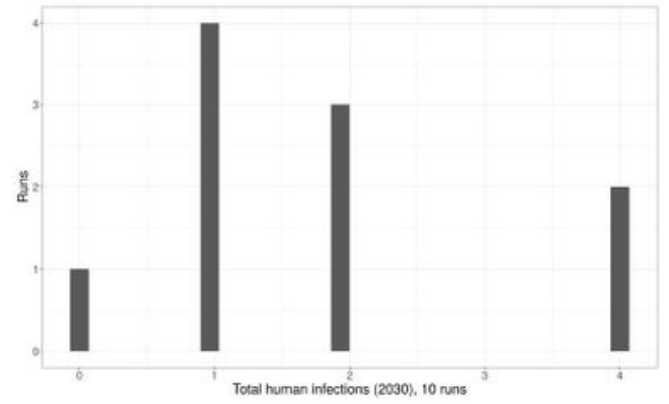

(d) $75 \%$ ITC

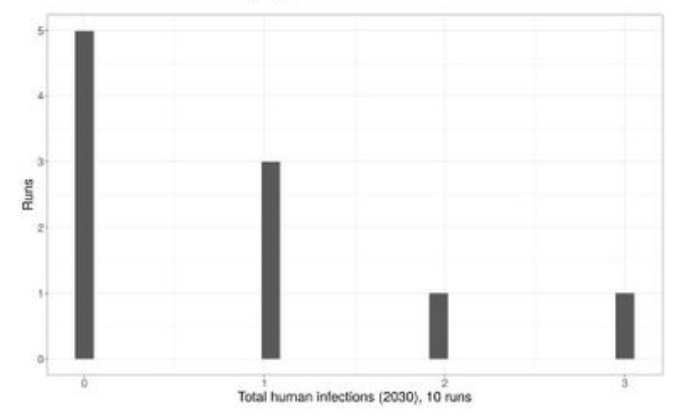

(f) $100 \% \mathrm{TT}, 100 \%$ ITC

Figure 5: Predicted cases in 2030, Malawi, over 10 runs. ITC: insecticide treatment of cattle and pigs; TT: trypanocide treatment of cattle and pigs. TT coverage assumed to be $50 \%$ unless otherwise specified.

\section{Figure 5}

Please See image above for figure legend. 


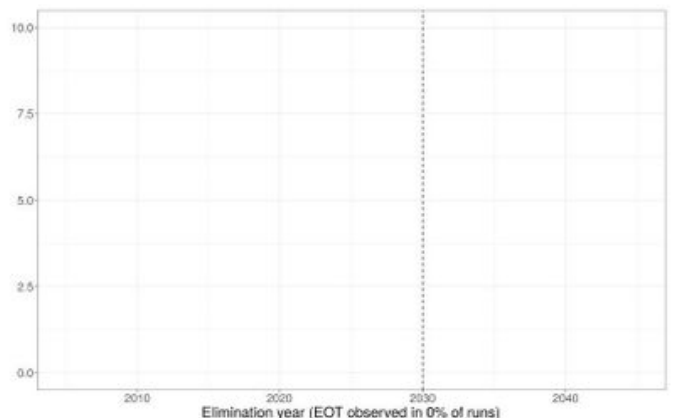

(a) Base case

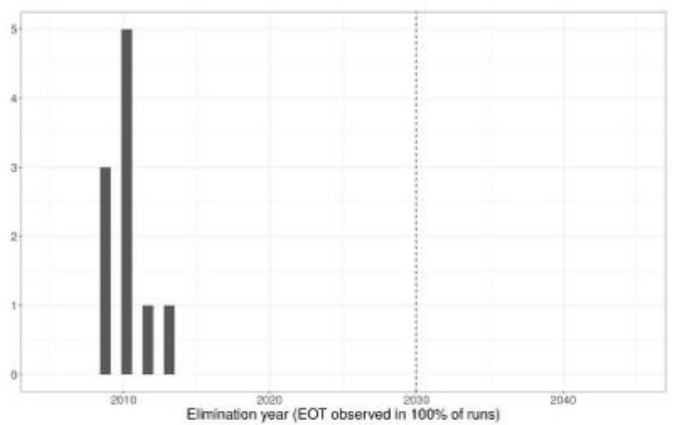

(c) $25 \%$ ITC

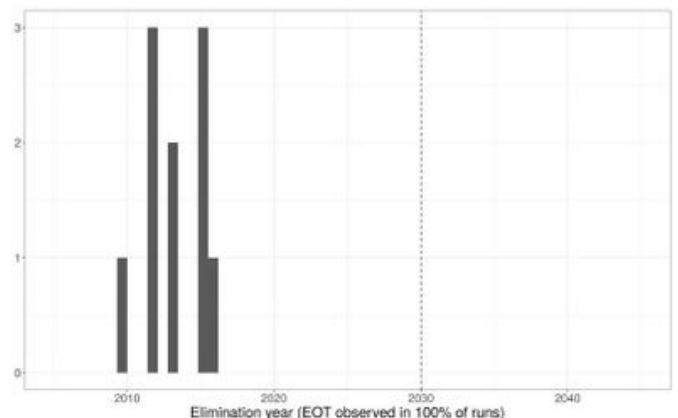

(b) $12.5 \%$ ITC

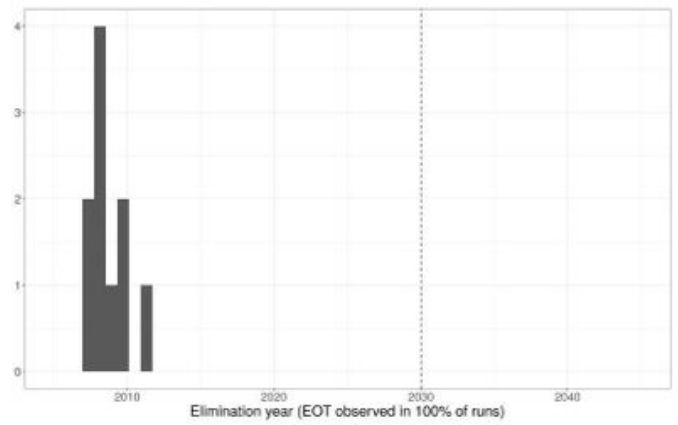

(d) $50 \%$ ITC

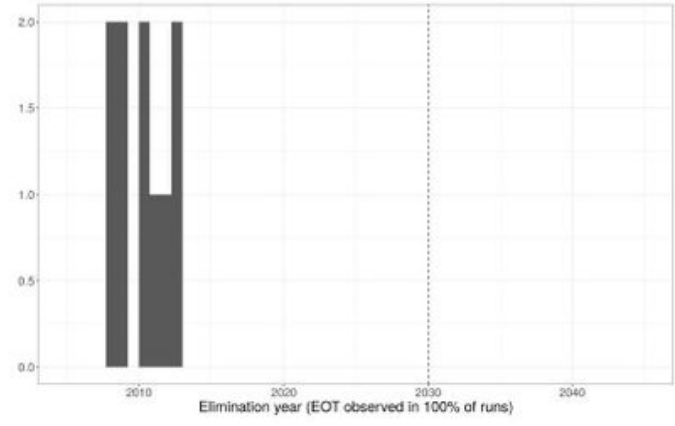

(e) $25 \% \mathrm{TT}, 25 \%$ ITC

Figure 6: Predicted year of gHAT elimination, Uganda, over 10 runs. ITC: insecticide treatment of pigs; TT: trypanocide treatment of pigs. TT coverage assumed to be $0 \%$ unless otherwise specified. Dashed vertical line marks 2030 (WHO target year for gHAT EOT)

\section{Figure 6}

Please See image above for figure legend. 


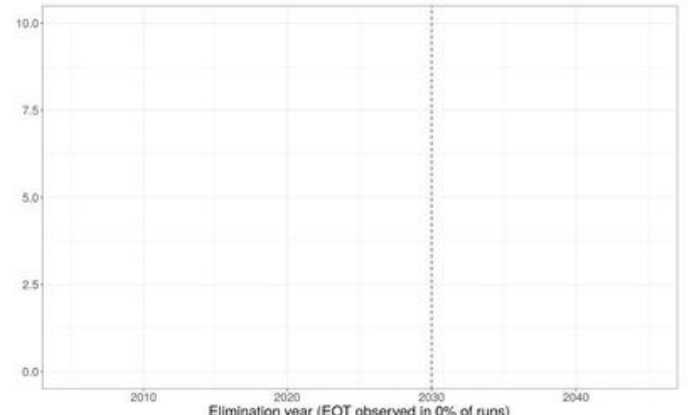

(a) Base case

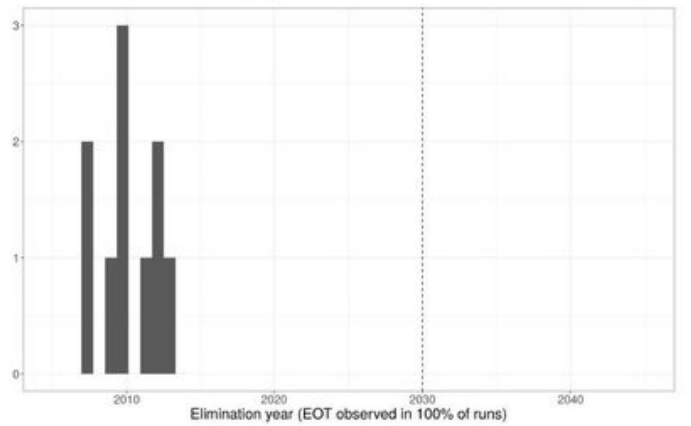

(c) $25 \%$ ITC

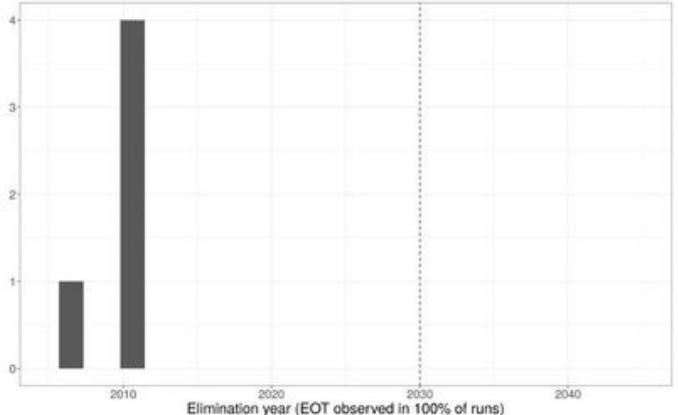

(b) $12.5 \%$ ITC

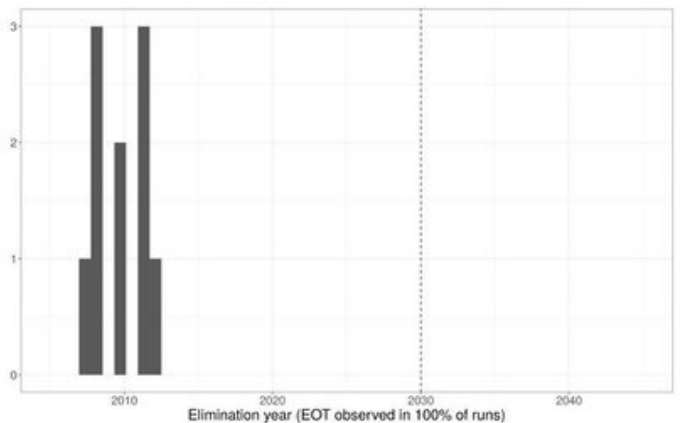

(d) $50 \%$ ITC

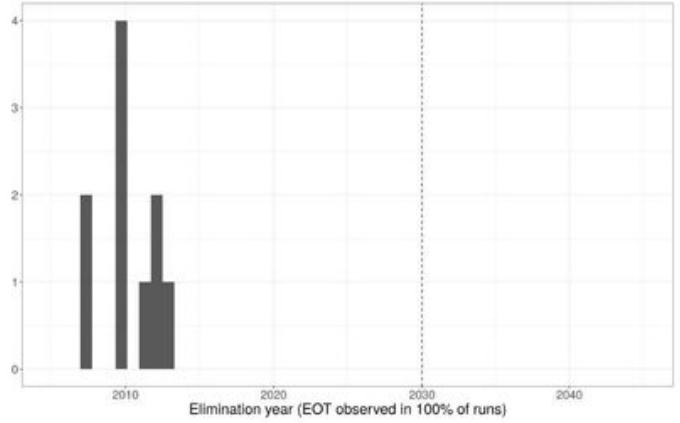

(e) $25 \% \mathrm{TT}, 25 \%$ ITC

Figure 7: Predicted year of gHAT elimination, DRC Equateur Nord focus, over 10 runs. ITC: insecticide treatment of pigs; TT: trypanocide treatment of pigs. TT coverage assumed to be $0 \%$ unless otherwise specified. Dashed vertical line marks 2030 (WHO target year for gHAT EOT)

\section{Figure 7}

Please See image above for figure legend. 


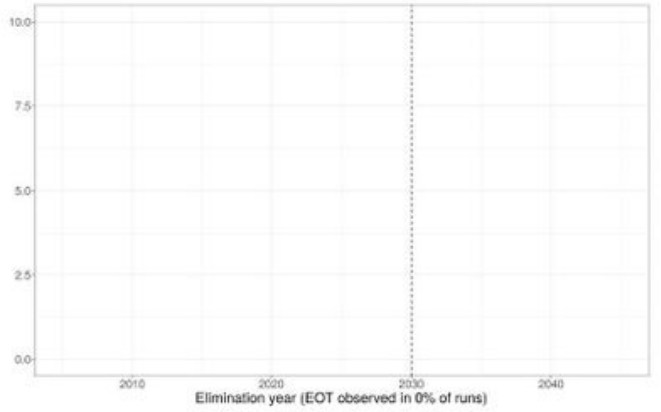

(a) Base case

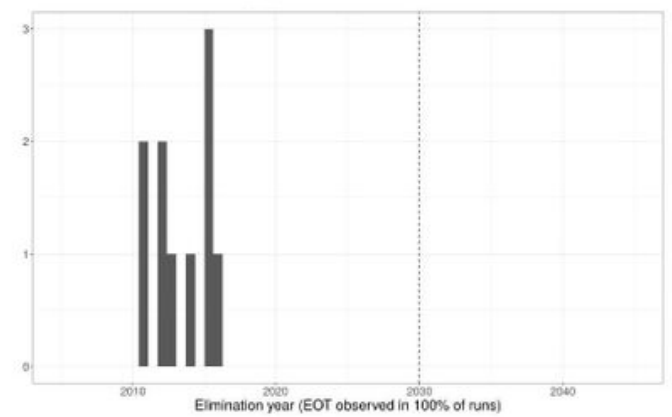

(c) $25 \%$ ITC

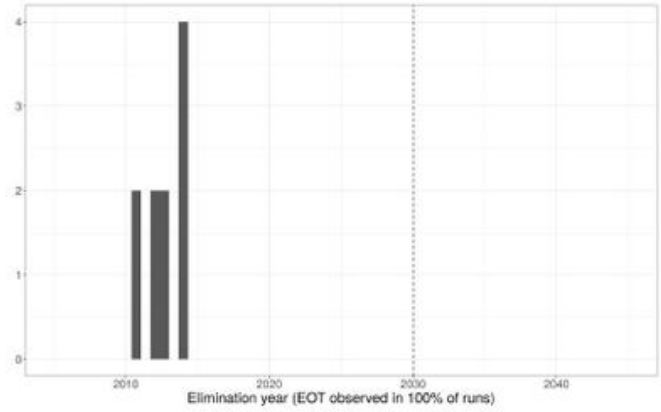

(b) $12.5 \%$ ITC

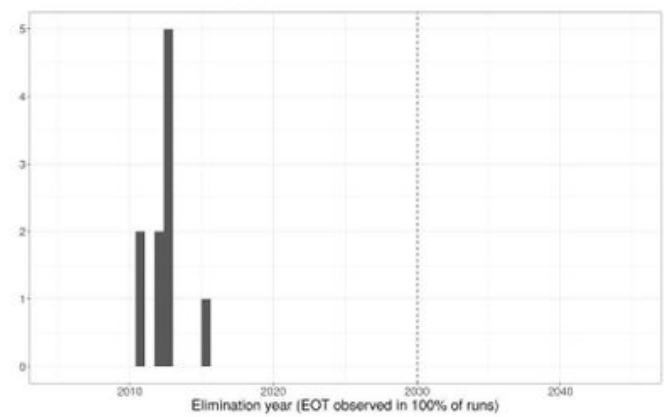

(d) $50 \%$ ITC

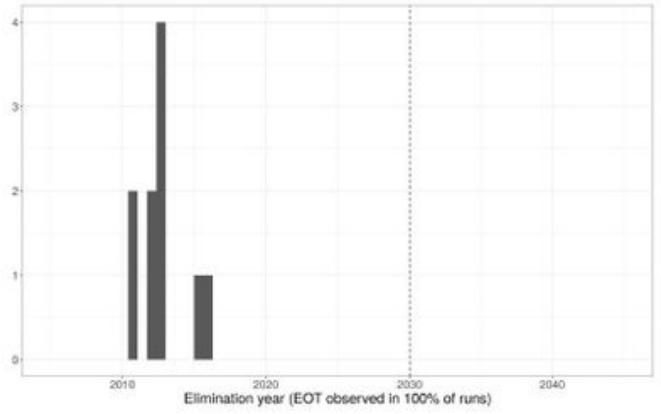

(e) $25 \% \mathrm{TT}, 25 \%$ ITC

Figure 8: Predicted year of gHAT elimination, DRC Bandundu/Sakuru foci, over 10 runs. ITC: insecticide treatment of pigs; TT: trypanocide treatment of pigs. TT coverage assumed to be $0 \%$ unless otherwise specified. Dashed vertical line marks 2030 (WHO target year for gHAT EOT)

\section{Figure 8}

Please See image above for figure legend. 


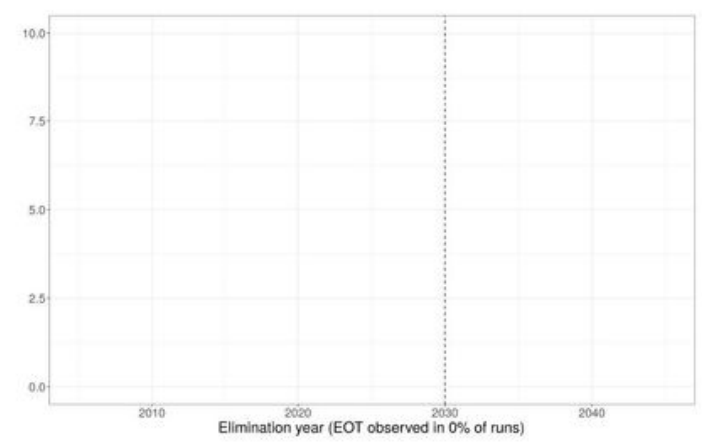

(a) Base case

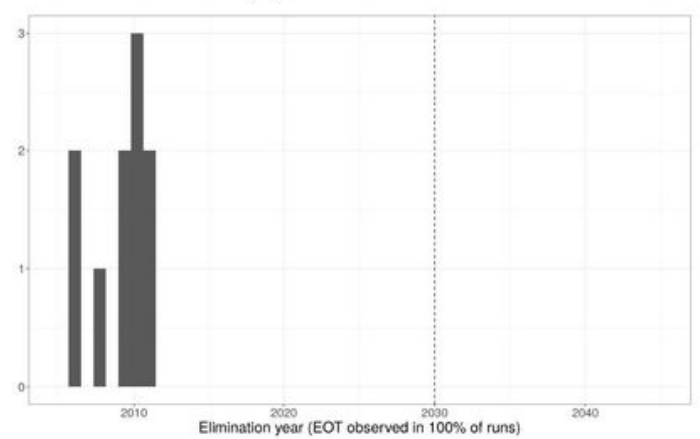

(c) $25 \%$ ITC

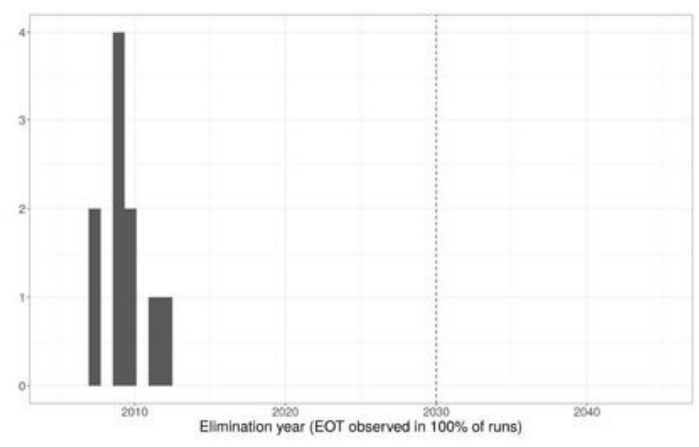

(b) $12.5 \%$ ITC

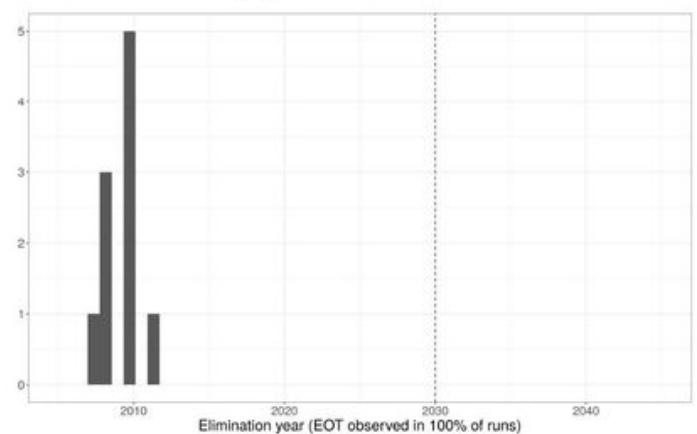

(d) $50 \%$ ITC

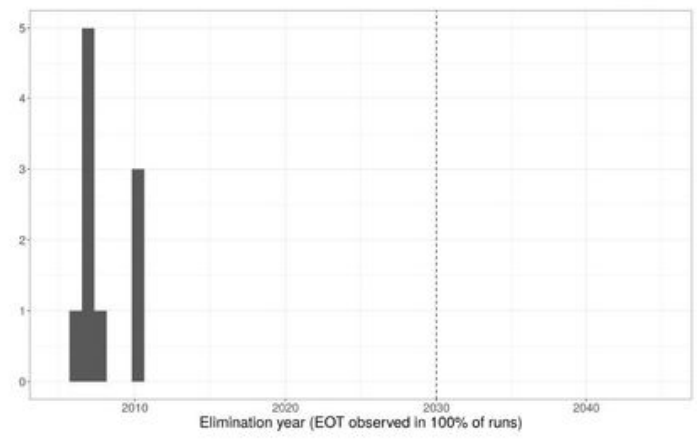

(e) $25 \%$ TT, $25 \%$ ITC

Figure 9: Predicted year of gHAT elimination, South Sudan, over 10 runs. ITC: insecticide treatment of pigs; TT: trypanocide treatment of pigs. TT coverage assumed to be $0 \%$ unless otherwise specified. Dashed vertical line marks 2030 (WHO target year for gHAT EOT)

\section{Figure 9}

Please See image above for figure legend.

\section{Supplementary Files}

This is a list of supplementary files associated with this preprint. Click to download. 
- Additionalfile1Modelparameters.pdf

- Additionalfile2Modelequations.pdf

- Additionalfile3Modeltrajectories.pdf

- Additionalfile4Livestockmaps.pdf

- Modeldiagram.png 